\title{
How to run an induction meeting for house officers
}

\author{
Rodney Gale, Gordon Jackson, Michael Nicholls
}

This article is based on the experiences of the authors in designing and running induction courses at Lewisham Hospital. The induction process was studied as a component in a counselling, monitoring, and support system for doctors in training. Our methods are based on needs assessments of three separate cohorts of house officers and have been refined through continuous feedback and evaluation.

\section{Induction: why bother?}

The costs of induction are manifested in a loss of service cover from house officers during the meeting and some extra work for the clinical tutor and staff of the postgraduate medical centre. We see induction as a responsibility of the clinical tutor on behalf of the consultants who are educational supervisors.

The benefits come from integrating the new house officers as quickly as possible into the local systems and from reducing their anxiety to allow them to feel, and be, more competent more quickly. The pressures and responsibilities on all other team members are also reduced by efficient and appropriate induction.

\section{Philosophy of induction}

Below are listed the elements of the philosophy of induction at Lewisham.

(1) General induction focuses on the needs and perceptions of the new house officers and not on the desires of the organisation. It should take place at the very start of the new appointment.

(2) Induction based on the firm will be needed to supplement the general induction and can take place over a longer time.

(3) Attendance needs to be compulsory and must not be prevented by other arrangements.

(4) The meeting should be "user friendly" and free from interruptions from bleeps.

Many current induction meetings consist of a collection of departmental heads or specialty tutors turning up for a few minutes to pass on their greetings and a few words of wisdom. The new house officers may also be given highly detailed and directive information about some small area of their overall task. This process is perceived as berating the new recruits for the sins of their predecessors. We have discovered that information passed on in this form, to a group of people who are preoccupied with the details of their forthcoming duties, is not absorbed and not remembered. It amounts to a waste of everyone's time to run an induction meeting in the classic way.

\section{South East Thames \\ Regional Health Authority, London WCIN 3EJ \\ Michael Nicholls, postgraduate dean}

Correspondence to: Dr Jackson.

BMF 1992;304:1619-20
The model we suggest is best illustrated through a detailed presentation of a typical timetable with supporting notes, as follows. The induction process for the August intake consists of a full day of activities that take place on 31 July. The invitations are sent out as soon as the names of the new recruits are known so that holidays or summer trips can be coordinated. The house officers are paid for their time, currently from soft monies, but this is soon to be part of an employment contract.

There are huge advantages in holding the meeting on 31 July:

- The departing house officers are still in post and carrying the bleeps

- The new recruits can meet the patients and meet their firms, without having to carry the service workload

- Shadowing the current postholder is an efficient way to learn the duties

- A more relaxed pace can be adopted with more involvement from the new recruits

- There may be some difficulties to resolve over accommodation and luggage.

\section{TIMETABLE AND NOTES}

0830-0900 Assemble and breakfast

A breakfast of coffee and croissants is provided. Good food and a chance to meet old friends help to set the welcoming and friendly tone.

0900-0915 Welcome from the clinical tutor

An overview of the hospital, an introduction to the educational aspects of the job, and some thoughts on career moves are presented.

\section{5-0930 Meet the centre manager}

An opportunity to meet a friendly person who will provide support and concrete help with job applications and curriculum vitaes. Formal educational opportunities are also described.

\section{0-1030 Being a house officer at Lewisham}

This session is run as parallel discussions in small groups. There are two groups for general medicine and one each for general surgery and orthopaedics. We originally used two groups of nine but found the size of the groups inhibited participation. The discussions are each led by one of the current house officers. Their brief is to describe what it is actually like to be a house officer at Lewisham and to answer questions that the new recruits wish to raise. This session is the most important in the induction process, as subsequent inquiries of participants have disclosed. It is essential to use a house officer as group leader to minimise hierarchical distance between the group members. The session is generally animated and vocal and is one where it is possible to air very basic concerns without loss of face. The clinical tutor exercises no control over the content or conduct of the small groups. We consider that the departing house officers understand hospital procedures and are in the best position to communicate them. The alternative method of having the most senior person from a department or specialty to put across "the party line" has been shown to be ineffective at a time when more fundamental anxieties 
Learning the ropes: doctors starting first house officer jobs are encouraged to shadow their predecessors

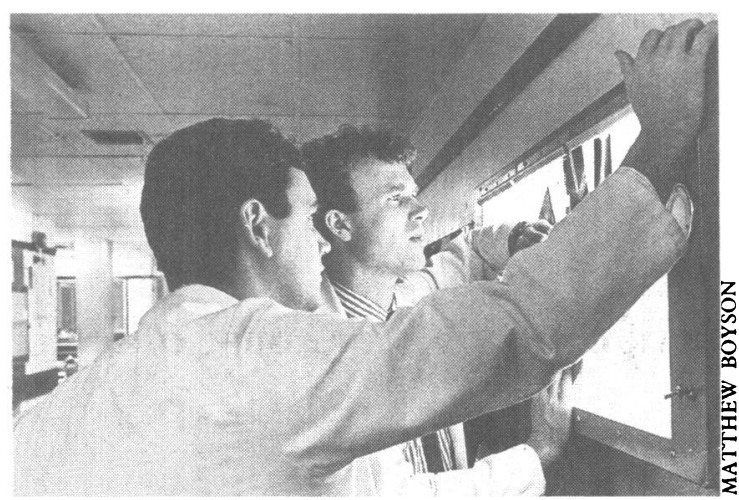

about the detailed nature of their duties are uppermost in the prospective house officers' minds. This is not to deny the importance of the information but to suggest there may be better ways to communicate it.

\section{0-1045 Coffee}

\section{5-1115 Meeting death}

This section reinforces the procedures for recognising and certifying death and covers the procedures for postmortem examinations and referrals to the coroner.

\section{5-1200 Breaking bad news}

A revisiting of principles learnt in medical school, conducted by a consultant with a particular interest in communications. Our research has disclosed that dealing with death and breaking bad news are major concerns of the new house officers.

\section{0-1230 Counselling, monitoring, and support}

After the bad news, some good news concerning what the house officers can expect in terms of feedback, support, advice, and encouragement from their supervising consultants and from the medical centre.

\section{0-1330 Lunch}

\section{0-1700 Meet the firm}

We arrange for house officers to be met at lunchtime by the person they will replace, or by another doctor from the firm. We encourage all firms to produce a couple of pages of notes to list the main duties of the house officer and to explain the timetable. The afternoon is spent meeting the patients and learning the systems used on the relevant firm.

\section{House officers' handbook}

A very important part of the induction process is the handbook. Almost every hospital has a handbook of some form. Often the handbooks are produced by management, for management and they contain a vast amount of detail about employment, conditions of work, health and safety, and hospital policy on everything. Our research shows that these large handbooks tend to be seldom used and easily misplaced. Our handbook is small and slim so that it fits easily in the pocket of the doctor's white coat. It contains information considered essential by the house officers themselves. Editorial control rests with a group of four house officers who take on the responsibility of refining the handbook every six months. The clinical tutor has a page on the counselling scheme and another on dealing with the coroner; otherwise the content is the choice and responsibility of the house officers. We think this is an important part of establishing a friendly and trusting atmosphere in which house officers can realise their full potential.

We believe that the handbook must remain small and short if it is to be used. There is little point in producing a wonderfully complete textbook of hospital life and clinical procedures if it is not going to live in doctors' coat pockets and be a constant companion. (We are not opposed to general information about hospital policy and procedure being available, but it should not be included in the house officers' pocket book.)

\section{February intake}

We use an entirely different procedure for the 1 February intake. It would be technically possible to assemble the new recruits on 31 January, but only at the cost of removing them from their duties elsewhere. We believe that such a process is unnecessary as most people are starting their second house job. The main issue is making the switch from medicine to surgery and orthopaedics and vice versa; the overwhelming anxieties about being a house officer at all, are a thing of the past.

Our induction process takes place on the first morning and lasts about one and a half hours. We arrange for internal cover so that a "bleep free" environment can be created. The bleeps are held and answered by the centre manager. The meeting has the following components:

- Breakfast and handbooks to browse through

- Welcome from the clinical tutor and centre manager

- Small group discussions on being a surgical or medical house officer

- The counselling, monitoring, and support scheme

- Collection by a member of the firm, when possible.

There are no house officers available to lead the small group discussions, so the next best choice is a senior house officer. In practice, the choice is dictated by the need to maintain the service while the house officers are engaged on the induction course.

In many hospitals one or two people will be starting their first house officer job on 1 February. We aim at encouraging such people to come to the hospital and shadow their predecessor for one or two days at the end of January. We also ensure that their consultants are aware they are new starters.

An informal system already exists, in which prospective house officers contact their predecessors during January. We have tried to build on this system by giving our incumbent house officers the names and contact numbers of their replacements so that they can initiate the arrangements for an informal visit.

\section{Evaluation}

We have now evaluated two such induction meetings. The house officers remember the event and the contents of the meeting. They are unable to think of any additional matters to include, except some specific revision of cardiopulmonary resuscitation or trauma management, and can think of nothing that should not be included in the induction meeting. This is in contrast to our evaluation of a classic induction meeting, consisting of three and a half hours of talks, with minimal discussion and interaction, where one month afterwards only one of 12 house officers could recall any of the content and three could not recall having been at the meeting.

In conclusion, we strongly recommend adoption of a user friendly, participative style of general induction meeting that addresses the real concerns of the house officers themselves. We urge recognition that further induction to individual firms and their practices will be needed. The general induction meeting starts the process but does not finish it.

(Accepted 15 May 1992) 